\title{
Retraction Note to: New Algorithms for Solving RFMLrR Circulant Linear Systems in Information
}

\author{
Jianhua Liu, Dengjie Wu, and Zhao-Lin Jiang \\ Department of Mathematics, Linyi University, Linyi 276005, P.R. China \\ jhliu@1yu.edu.cn, wdj20130313@163.com, \\ jzh1208@sina.com
}

Retraction Note to:

Chapter "New Algorithms for Solving RFMLrR

Circulant Linear Systems in Information" in:

Y. Yang, M. Ma, and B. Liu (Eds.):

Information Computing and Applications, CCIS,

DOI: 10.1007/978-3-642-53932-9_17

The paper starting on page 169 of this publication has been retracted because it does not differ sufficiently from the paper starting on page 59 of the same publication. One of the authors is the same.

The updated original online version for this chapter can be found at DOI: 10.1007/978-3-642-53932-9_17 